\title{
THE FEASIBILITY OF INTEGRATING ALCOHOL RISK-REDUCTION COUNSELING INTO EXISTING VCT SERVICES IN KENYA
}

\author{
Caroline Mackenzie*1, Karusa Kiragu ${ }^{1}$, George Odingo ${ }^{1}$, Rukia Yassin ${ }^{2}$, Peter \\ Shikuku², Patrick Angala², William Sinkele ${ }^{3}$, Melania Akinyi ${ }^{4}$ and Nduku Kilonzo ${ }^{2}$ \\ ${ }^{1}$ Population Council/Horizons, Kenya, \\ ${ }^{2}$ Liverpool VCT, Care and Treatment, Kenya, \\ ${ }^{3}$ Support for Addiction Prevention and Treatment Africa Trust, Kenya, \\ ${ }^{4}$ The Steadman Group, Kenya
}

\begin{abstract}
This pretest-posttest separate-sample study with intervention and comparison groups documented the abilities and willingness of trained voluntary counseling and testing (VCT) service providers to integrate alcohol screening and risk reduction counseling into their routine service delivery. Pre-test $(\mathrm{n}=1073)$ and post-test data $(\mathrm{n}=1058)$ were collected from different clients exiting from $25 \mathrm{VCT}$ centers. A 12-month intervention that required all VCT providers from the intervention groups to screen all VCT clients for their alcohol use and offer them brief risk reduction alcoholrelated counseling was implemented. At post-test, the intervention group clients $(n=456)$ had better study outcomes than the comparison group clients $(n=602)$. Intervention clients were more likely to report that their VCT service provider had: asked them about their alcohol use $(83 \% \mathrm{vs}$. 41\%: $p<0.05)$; asked them about their partner's alcohol use ( $72 \%$ vs. $22 \%)$; screened them for their alcohol use $(77 \%$ vs. $33 \%: \mathrm{p}<0.05)$; and gave them feedback about their screening results $(67 \%$ vs. $35 \%$ : $\mathrm{p}<0.05)$. The study concluded that VCT service providers are able and willing to integrate alcohol risk reduction services during routine VCT services, and supports the integration of alcohol risk reduction counseling at VCT services in Kenya.
\end{abstract}

KeY Words: Alcohol, VCT, Integration, Service provision, Risk reduction, Counseling, Kenya

\section{INTRODUCTION}

Alcohol use has been identified as one of the underlying social factors that drive HIV risk behavior. The association between alcohol use, reduced sexual inhibitions, HIV transmission and individual behavior has been demonstrated in many studies in both developing and developed countries. Weiser et al. (2006), Zablotska et al. (2006), Morojele et al. (2005), and Shaffer et al. (2004) have all documented that alcohol is thought to fuel HIV transmission by blunting one's behavioral self-monitoring and increasing the likelihood of multiple partners, unprotected sex, intergenerational sex, and commercial sex.

Corresponding Author: Caroline Mackenzie*, P. O. Box 45 - 00502. Karen, Kenya; Email: nzilanimackenzie@yahoo.com Telephone: 254721458625 
Despite the recognition of the role that alcohol plays in fuelling the HIV epidemic, programmers have been faced with the challenge of how to respond to alcohol use in HIV prevention. In sub-Saharan Africa, few HIV prevention programs, including voluntary counseling and testing (VCT) programs have directly addressed the issue of HIV risk behavior as a result of alcohol use. This is partly because service providers offering HIV prevention programs are not formally required to ask their clients about whether they use alcohol, hence missing a great opportunity to address this risk factor with their clients.

In recognition of this gap in service provision, in 2005, the WHO requested member states to intensify efforts to reduce the burden of alcohol-related problems nationally, regionally and globally (WHO, 2005). A policy paper presented to a Regional Health Ministers Conference in Kenya, on February 2006 urged member states to put issues related to alcohol in their national HIV/AIDS strategies and ensure that appropriate alcohol and HIV/AIDS policies and programs were in place (Morris et al., 2006).

To respond to this, in Kenya, VCT programs were identified as one of the key entry points for services designed to reduce HIV risks associated with alcohol use. In 2007, Kenya had over 900 VCT centers (LVCT, 2007), and so they were considered an important entry point for targeting risk reduction messages to VCT clients who use alcohol. During pre-test counseling, clients are given information on modes of HIV transmission and triggers of risky behavior. Thus, the VCT setting offers an optimal venue for discussing alcohol as a factor in HIV transmission, and for helping clients formulate risk-reduction plans. However, because greater quantities of alcohol often predict greater sexual risks, it is important for service providers to screen clients for their levels of alcohol use so as to offer relevant advice and referral. Also, because men are more likely to drink and engage in risky behavior, whereas women's risks are often associated with their male sexual partner's drinking (Kalichman et al., 2007), it is important for peo- ple to be screened for both their own alcohol use and that of their partners, and appropriate messages and advice offered.

To respond to these issues, researchers from Kenya designed a study whose objective was to determine the feasibility of integrating alcohol risk reduction counseling into VCT service provision. This paper describes the study project and its findings.

\section{METHOD}

\section{Study Design}

This study employed a pre-test post-test research design with separate samples, and two treatment groups (intervention and comparison). Pre-test and post-test data were collected from VCT clients in 15 static and 10 mobile VCT sites from three provinces in Kenya. The three provinces were purposively selected because that is where the intervention partner, Liverpool VCT, Care and Treatment (LVCT), had VCT centers that they were supporting. The sampled VCT centers were purposively selected because they were situated in high density population areas with anecdotal evidence of high alcohol use. Eight of the static sites were randomly allocated to the intervention group, while the remaining seven, together with all the mobile VCT sites, were allocated to the comparison group. Because providers at the mobile sites move from one site to another, all of these sites needed to be assigned to the same treatment group. This decision to allocate al the mobile sites to the comparison group was made randomly.

\section{Pre- and Post-Test Data Collection}

In June 2006, the pre-test data were collected using face-to-face exit interviews from 1,073 VCT clients from all the participating VCT centers. In July 2007, after a year of implementing intervention activities, the posttest study was conducted among 1,058 VCT clients. Although the same facilities were visited, different clients were interviewed. Sample sizes at both pre-test and post-test allowed for 
a $95 \%$ confidence level and a power of $90 \%$. At both study points, the same data collection methods and tools were applied. The respondent's drinking levels were assessed using the Alcohol Use Disorders Identification Test [AUDIT] (Babor et al., 2001; Saunders 1993). The data collection tool was a structured questionnaire that had been pre-tested and translated into two local languages, and at post-test had an additional section that measured client's exposure to the intervention. Interviewers were trained on administration of the survey questionnaire, the informed consent form, and observation of ethical procedures (described further below). Each interviewer was stationed at the VCT center until they reached the allocated sample for that VCT center, which had been calculated to be proportional to the client flow. Potential respondents were invited for the interview after they had completed their VCT session. The eligibility criteria were that respondents were 18 years and above, had given written consent, and had gone through the VCT session, irrespective of whether they took the HIV test or not.

\section{Description of the Intervention}

To respond to VCT service provider gaps, the study aimed to establish whether trained VCT providers would be able and willing to integrate alcohol risk reduction counseling into their routine service delivery. The intervention partners were two local nongovernmental organizations. LVCT, which offers training, research and support for VCT counselors, and Support for Addiction Prevention and Treatment in Africa Trust (SAPTA), which offers training for drug and substance abuse counselors. Staff from both LVCT and SAPTA, with technical support from Population Council, developed a three day alcohol training module to integrate alcohol risk reduction counseling into VCT services. Titled "Integrating alcohol risk reduction counseling into VCT services: training module for VCT counselors", this module has 15 units that cover types of substances, definitions, reasons for use, effects on the body, relationship with risks for HIV, protocol for integrating alcohol screening and counseling into VCT centers, and treatment for excessive users of alcohol. In September 2006, this alcohol module was used to train $32 \mathrm{VCT}$ services providers from the eight $\mathrm{VCT}$ centers in the intervention group. The key activities VCT providers were expected to integrate into their VCT counseling sessions included:

a) Screening clients for their alcohol use and that of their partners: During regular HIV risk assessment, the VCT providers were trained to screen clients for their alcohol use. They used the CAGE screening tool (Ewing, 1984) that focuses on 4 questions asked to each client to screen for alcohol use levels.

1) Have you ever felt that you need to $\underline{\mathrm{Cut}}$ down on your drinking?

2) Have people Annoyed you by criticizing your drinking?

3) Have you ever felt bad or Guilty about your drinking?

4) Have you ever had a drink first thing in the morning (Eye-opener) to steady your nerves, e.g. get rid of a hangover or get your day started?

This tool was modified to also screen clients for their partners' alcohol use. The same four questions were used to ask about their partners, i.e. has your partner even felt that they needed to cut down on their drinking, and so on. Two or more "Yes" responses were indicative that the client (or their partner) has problems with alcohol. Following this screening, those with an alcohol problem were offered the following brief intervention.

b) Brief intervention for alcohol users: This was modeled based on a similar one from WHO (Babor et al., 2001; NIAAA, 2005). It was a client-centered, 5-10 minutes intervention where the provider explained the meaning of the screening, and gave the client feedback from the screening results. Clients who were not currently taking alcohol were congratulated and encouraged to continue abstaining. Clients with a positive CAGE were empathized with and encouraged to take responsibility 
for their possibly risky drinking behavior and given clear, explicit advice and information on how to reduce/stop their drinking and explore with their partner the possibility for alcohol reduction. A menu of alternate strategies for tackling alcohol problems was offered. This included setting of realistic and attainable goals, improving self esteem and self belief that aimed to empower the clients to change their behavior, assured them that they have the capability to change, and solicited their commitment to do so.

c) Referrals to treatment and care services for clients with alcohol problems: During the training, providers were given a referral list for alcohol treatment centers and Alcoholics Anonymous (AA) meetings. They used these lists to refer clients who needed alcohol related post-test care and support.

d) Provision of educational materials: To supplement the information that the clients were receiving from the providers, existing brochures and posters that sought to educate and offer client information on the dangers of excessive use of alcohol were reprinted and distributed to the intervention group. These posters hang at conspicuous places in all the VCT centers at the intervention group.

\section{Additional Data Collection Activities at the Intervention Sites}

Three focus group discussions (FGDs) with providers and three with clients were conducted before the intervention to determine whether the program would be acceptable to them. Further, the researchers sought to document the amount of time that the screening and discussions on alcohol were taking. During the intervention activities, an interviewer visited four randomly selected VCT centers from the intervention group. The interviewer sat outside the VCT room and recorded the amount of time each VCT session took. In addition, after each VCT session, the provider was asked to estimate the amount of time they had taken discussing alcohol issues. A total of 32 observations and interviews were documented.
Ethical Considerations and Confidentiality

The study protocol and data collection tools had been reviewed and approved by Population Council's and Kenyatta National Hospital's ethical review committees. All respondents were informed about the study using an informed consent form, which they read or were read to. This form informed respondents about the study, about their voluntary participation without fear or retribution, the benefits and risks of participating in the study, and gave contact details of the principal investigators. All respondents gave written consent by signing the consent form. The signed form was separated from the data collection forms. Respondents who were distressed, for example, as a result of a positive HIV diagnosis, were not interviewed. Respondents were not required to reveal their HIV status to the interviewer and they were not asked about their HIV status. Interviews were conducted in a private place and no identifying information was recorded on the questionnaire. All data collected were kept confidential, and presented in aggregate terms to prevent deductive disclosure. Individual responses were only disclosed to those directly involved in activities associated with data collection, entry, or analysis.

\section{Data Management and Analysis}

To test whether the intervention was feasible, researchers focused on the quantitative post-test data set, and compared differences in outcomes between the intervention and comparison groups. The following variables of interest were measured: whether the provider had asked the client about their alcohol use, and that of their partner; whether the provider had screened clients for their levels of alcohol use; whether the providers gave the clients feedback or the results of the screening; whether the providers had referred clients with alcohol problems for further alcohol counseling; whether the clients had been exposed to alcohol education materials. The qualitative FGDs measured whether the alcohol intervention at VCT centers was acceptable to both clients and providers. The qualitative data were 
transcribed and verbatim quotes used to describe outcomes.

Quantitative data from the exit interviews were entered through scanning of the completed questionnaires. Formic software, a new technology that ensures speed and accuracy of data entry was used. To verify the scanned data, $15 \%$ of the questionnaires were rescanned and $10 \%$ were physically checked for consistencies. Data were then cleaned and analyzed using SPSS version 13.0 and STATA version. 9.0. Frequencies and bivariate analysis provided descriptive summaries and comparisons between the variables of interest and treatment group. Tests of significance were carried out using Pearson's chi-square test of independence. Results were considered statistically significant at or below a p-value of 0.05 .

\section{RESULTS}

\section{Demographic Characteristics of the Study Population}

Respondents from both the intervention and comparison groups had almost similar demographic characteristics. Apart from the post-test sample among the comparison group that had $43 \%$ of the respondents being male, the rest of the samples had over $50 \%$ of the respondents being male (see Table 1). Over $60 \%$ of the respondents were below the mean age of 29 years, over $50 \%$ were single (never married), over $40 \%$ had a secondary school education and above, and over 59\% were Protestants. Over three quarters of the respondents had a sexual partner. About $20 \%$ of the respondents were current users of alcohol.

Table 1: Demographic characteristics of the study population at pre-test and post-test, by intervention and comparison groups

\begin{tabular}{|c|c|c|c|c|}
\hline \multirow[t]{2}{*}{ Characteristic } & \multicolumn{2}{|c|}{ Intervention group } & \multicolumn{2}{|c|}{ Comparison group } \\
\hline & $\begin{array}{c}\text { Pre-test } \\
n=483\end{array}$ & $\begin{array}{c}\begin{array}{c}\text { Post-test } \\
\mathrm{n}=456\end{array} \\
\text {. }\end{array}$ & $\begin{array}{c}\text { Pre-test } \\
\mathbf{n}=590\end{array}$ & $\begin{array}{c}\begin{array}{c}\text { Post-test } \\
n=602\end{array}\end{array}$ \\
\hline \multicolumn{5}{|l|}{$\operatorname{Sex}(\%)$} \\
\hline Male & 54 & $56^{* *}$ & 51 & $43 * *$ \\
\hline Female & 46 & $44 * *$ & 49 & $57 * *$ \\
\hline \multicolumn{5}{|l|}{ Age in years } \\
\hline Below mean* & 64 & 69 & 65 & 66 \\
\hline Above mean & 36 & 31 & 35 & 34 \\
\hline \multicolumn{5}{|l|}{ Marital status (\%) } \\
\hline Singe (never married) & 57 & 63 & 52 & 55 \\
\hline Married & 33 & 33 & 39 & 37 \\
\hline Divorced/separated/widowed & 10 & 5 & 9 & 8 \\
\hline \multicolumn{5}{|l|}{ Education level (\%) } \\
\hline Primary or less & 32 & 27 & 42 & 44 \\
\hline Secondary & 42 & 47 & 44 & 43 \\
\hline University and above & 11 & 6 & 2 & 2 \\
\hline Other formal & 15 & 20 & 11 & 11 \\
\hline \multicolumn{5}{|l|}{ Religion (\%) } \\
\hline Protestant & 67 & 59 & 62 & 64 \\
\hline Catholic & 24 & 32 & 31 & 27 \\
\hline Muslim & 6 & 8 & 3 & 2 \\
\hline \multicolumn{5}{|l|}{ Alcohol use (\%) } \\
\hline Current drinkers & 22 & 27 & 20 & 20 \\
\hline Used to drink but stopped & 43 & 40 & 44 & 45 \\
\hline Never drank & 35 & 32 & 35 & 35 \\
\hline
\end{tabular}

*Mean $=29$ years

$* * p<0.05$. Because all mobile sites were allocated to the comparison group, these differences could reflect seasonal variations, market days, and other factors that influence gender based activities. 


\section{Results of the Individual Variables of Interest of the Post-Test Sample Only, Comparing the Intervention and Comparison Group}

Proportions of respondents asked by providers about their alcohol use: At post-test, 83\% of the respondents at the intervention group $(n=456)$ reported that they had been asked whether they use alcohol, compared to $41 \%$ of respondents at the comparison group $(\mathrm{n}=602)$ $[p<0.05]$.

Proportion of respondents asked by providers about their partner's alcohol use: At posttest, among respondents with partners, $72 \%$ of them from the intervention group $(n=365)$ reported that the VCT counselor had asked them whether their partners drink. At the comparison group $(n=442)$, only $22 \%$ reported the same $(p<0.05)$.

Proportion of respondents screened by providers for their alcohol use: All the respondents who were currently drinking alcohol and had disclosed this to the providers were asked whether the providers had asked them the four questions in the CAGE screening tool. At the intervention group ( $\mathrm{n}=91$ ), $77 \%$ of such respondents said that they had been screened, compared to only $33 \%$ at the comparison group $(\mathrm{n}=43)[p<0.05]$.

Proportion of respondents offered feedback by providers about the results of the screening: Among the respondents who were currently drinking alcohol and were screened for their levels of alcohol use, at the intervention group $(n=70), 47$ clients were given feedback of their screening results, compared to only five clients at the comparison group $(n=15)[p<0.05]$. In terms of the specific feedback given, at the intervention group, 33 of these clients $(n=47)$ were told that they had a drinking problem. These clients $(n=33)$ reported having been given the following advice (more than one answer was possible): 15 were asked to stop drinking; 12 were told the detrimental health effects of excessive drinking; 11 were informed that alcohol use could cause one to engage in risky sexual behavior, and 8 were told that they were drinking to harmful levels and that they needed to consider reducing their alcohol intake.
Description of the referrals offered by providers to alcohol abusers: All the current drinkers at the post-test survey who had been screened and told that they had a drinking problem were asked whether they had been offered any type of referral. Only data from the intervention group $(n=33)$ is given because the numbers from the comparison group were less than 15. Three clients reported that they were advised to go to an alcohol treatment center for further assessment by an addiction specialist. Another three were reported that they were advised to join an Alcoholics Anonymous (AA) group. Informal discussions with the service providers indicated that most clients wished not to be referred to alcohol treatment centers because of the high costs of services.

Proportion of clients exposed to alcohol education materials: All the clients at post-test survey were asked whether they had picked a brochure on alcohol from the facility to take home and read. At the intervention group $(n=456)$, only $8 \%$ of the clients had a brochure, while only $1 \%$ at the comparison group $(\mathrm{n}=602)[p<0.05]$. On being asked whether they had seen an alcohol poster at the VCT center, at the intervention group, $53 \%$ of the respondents said that they had seen one, compared to $16 \%$ of respondents at the comparison group.

Acceptability of the program among clients and providers: The program was acceptable to both clients and providers. Almost all the clients at both groups $(97 \%$ and $90 \%$ at the intervention and comparison group respectively) reported that they would have liked to have discussions about alcohol use while at the VCT center. During the FGDs, both clients and providers indicated that alcohol is a major risk factor for HIV risk, and that the VCT room was an appropriate place to help clients reduce their risks, as shown in this quote.

"Personally I feel the need for concern on the level of drinking... When you are assessing the risk... you will find that most of the clients will associate their risks on how they were drunk. You will find a client will say they did something because 
of how they drunk the other day. So I feel my need for concern of alcohol drinking in the VCT..."

VCT counselor, Nairobi.

Additional time taken to implement the alcohol intervention: The average time taken for the entire VCT session with the added alcohol component was 52 minutes (range: 46-90 minutes). The recommended time for VCT sessions is $45-60$ minutes. Providers estimated that the additional time taken by alcohol discussions was seven minutes. They did not find this additional time a burden.

\section{DISCUSSION}

The findings of this study suggest that it is feasible to integrate alcohol risk reduction counseling into VCT services in Kenya. The data showed that the intervention group had better study outcomes than the comparison group. Clients from the intervention group were more likely to have been asked about their own alcohol use and that of their partners. Clients from the intervention group, who were currently using alcohol were more likely to have been screened and given feedback about their drinking levels than similar clients at the comparison group. Clients with drinking problems reported having been advised to consider stopping or reducing their alcohol intake. They also reported having been advised about the dangers of excessive alcohol use, and the relationship between alcohol use and risky sexual behavior. These data indicate that trained VCT providers were able to integrate alcohol risk reduction counseling into VCT services. The alcohol counseling component increased the time taken during VCT sessions by an average of seven minutes, which providers did not find burdensome. Both providers and clients accepted the program and found the VCT room to be an appropriate venue for discussing alcohol related HIV risks. Hence, this study supports the integration of alcohol risk reduction counseling into VCT services in Kenya.
The findings indicate that the chances of a client having a brochure on alcohol on the index day were less than $10 \%$. This was mainly attributed to high demand of the materials. Hence, programs should ensure an adequate supply of information and education materials on alcohol consumption and its association with risky sexual behavior.

This study found that clients with alcohol problems were unwilling to be referred to alcohol treatment centers because of their high service costs. Service providers should devise innovative methods of ensuring clients with alcohol problems have access to continued support, for example through encouraging them to join AA group (which are free) or linking them up with community treatment centers that offer alcohol treatment services at more affordable costs.

This study used a separate sample pretestposttest research design. It was not a cohort study. As such, it was not designed to measure reduction in alcohol use or HIV risk behaviour that could be directly attributed to the intervention. Its main objective was to document whether trained VCT counselors could integrate alcohol screening and counseling into their regular service provision. Future studies could employ prospective research designs with clients who have been exposed to such an intervention, so as to document whether exposed clients would change their drinking behavior and related risks. Evidence of some intervention components at the comparison sites could be as a result of leakage of the intervention, through movement of service providers from one site to another, a factor which is difficult to control in such study designs.

\section{ACKNOWLEDGEMENTS}

The authors would like to thank Barry Levine, Rich Needle, Jennifer Brody, Teresa Secoli, Beatrice Ochieng', Elizabeth Njoki, Scott Kellerman, Ellen Weiss, Sam Kalibala, Naomi Rutenberg, Norah Omenda and Sherry Hutchinson for their various contributions towards this study and paper. Thanks to the research assistants, Carol Ngare, Dr. Anderson 
Kahindi, and the Medical Officers of Health for Nairobi City Council, Kasarani, Dagoretti and Mombasa Districts. Appreciation is extended to the management of: Kenyatta National Hospital, Mbagathi District Hospital, Coast Provincial General Hospital, National AIDS and STIs Co-ordinating Program, Family Health Options, Hope Worldwide, Eastern Deanery, and Shauri Moyo Baptist AIDS Response Unit. Thanks to FHI/IMPACT and PSI for their alcohol education materials. Thanks to all the clients and service providers who participated in the study. This study was funded by USAID/PEPFAR.

\section{REFERENCES}

Babor, T.F., Higgins-Biddle, J.C., Saunders, J.B., and Monteiro, M.G. (2001). The Alcohol Use Identification Test. Guidelines for use in primary care. Second Edition. Geneva: World Health Organization.

Ewing, J, A. (1984). Detecting alcoholism: The CAGE questionnaire. JAMA, 252, 1905-1907.

Patrick Angala, Data Manager. Number of VCT centres in Kenya in 2007. Liverpool VCT, Care and Treatment (2007). Verbal communication.

Kalichman, S.C., Simbayi, L.C., Kaufman M., Cain, D., and Jooste, S. (2007). Alcohol use and sexual risks for HIV/AIDS in sub-saharan Africa: Systematic review of empirical findings. Society of Prevention Research , 8, 141-151.

Morris, C.N., Levine, B., Goodridge, G., Luo, N., and Ashley, J. (2006). Three-country assessment of alcohol-HIV related policy and programmatic responses in Africa. African Journal of drug and Alcohol Studies, 5 (2), 169-184.

National Institute on Alcohol Abuse and Alcoholism [NIAAA] (2005). Helping patients who drink too much. A clinician's guide, 2005 edition. Washington, DC: U.S Department of Health and Human Sciences, National Institute of Health.

Morojele, N.K. and Kachieng'a, M. (2003). The development of a methodology of study factors to risky behaviour among alcohol users in diverse settings: Conceptual framework and instruments for qualitative and quantitative research of alcohol use related sexual risk behaviours in South Africa. A report prepared for the Department of Mental Health and Substance Dependence, WHO. Pretoria, South Africa.

Saunders, J. B, Aasland, O. G., Babor, T. et al. (1993). Development of the Alcohol Use Disorders Identification Test (AUDIT): WHO collaborative project on early detection of persons with harmful alcohol consumption - II. Addiction, 88,791-804

Shaffer, S.N., Njeri, R., Justice, A.C., et al. (2004). Alcohol abuse among patients with and without HIV infection attending public clinics in Western Kenya. East African Medical Journal, 81, 594-598.

Weiser, Sheri, D., Leiter, K., Heisler, M., McFarland, W., Percy-de Korte, F., DeMonner, S. M., Tlou, S., Phaladze, N., Iacopino, V and Bangsberg, D. (2006). A population-based study on alcohol and high risk sexual behaviours in Botswana. PloS Medicine, 3 (10). e392.

World Health Organization (2005). Alcohol use and sexual risk behavior: A cross cultural study in eight countries. Geneva, Switzerland.

Zablotska, Iryna. R, Gray, R.H., Serwadda, D., Nalugoda, F., Kigozi, G., Sewankambo, N., Lutalo, T., Mangen, F. W., and Wawer, M. (2006). Alcohol use before sex and HIV acquisition: a longitudinal study in Rakai Uganda. AIDS, 12, 20(8) 1191-1196. 